\title{
Real-World Experience with Benralizumab in Patients with Severe Eosinophilic Asthma: A Case Series
}

This article was published in the following Dove Press journal:

Journal of Asthma and Allergy

\section{Francesco Menzella $\mathbb{D}^{1}$ \\ Marco Bonavia ${ }^{2}$ \\ Matteo Bonini ${ }^{3}$ \\ Maria D'Amato ${ }^{4}$ \\ Salvatore Lombardo (iD ${ }^{5}$ \\ Nicola Murgia ${ }^{6}$ \\ Vincenzo Patella ${ }^{7,8}$ \\ Massimo Triggiani ${ }^{9}$ \\ Girolamo Pelaia (iD) ${ }^{10}$}

'Pneumology Unit, Arcispedale Santa Maria Nuova, Azienda USL di Reggio Emilia-IRCCS, Reggio Emilia, Italy;

${ }^{2}$ Pneumologia Riabilitativa - Ospedale Ge-

Arenzano, ASL3-, Genoa, Italy;

${ }^{3}$ Department of Cardiovascular and

Thoracic Sciences, Università Cattolica

del Sacro Cuore, Rome, Italy;

${ }^{4}$ Respiratory Department- Monaldi

Hospital AO Dei Colli, Naples, Italy;

${ }^{5}$ Pneumology Unit, Ospedale Giovanni

Paolo II, Lamezia Terme, Italy; ${ }^{6}$ Section of

Occupational Medicine, Respiratory

Diseases and Toxicology, University of

Perugia, Perugia, Italy; ${ }^{7}$ Division of Allergy

and Clinical Immunology, Department of

Medicine ASL Salerno, Santa Maria Della

Speranza Hospital, Salerno, Italy;

${ }^{8}$ Postgraduate Program in Allergy and

Clinical Immunology, University of Naples

Federico II, Naples, Italy; 'Division of

Allergy and Clinical Immunology,

University of Salerno, Fisciano, Italy;

${ }^{10}$ Department of Health Sciences,

Respiratory Unit, University "Magna

Graecia" of Catanzaro, Catanzaro, Italy

Correspondence: Francesco Menzella Pneumology Unit, Arcispedale Santa Maria Nuova, Azienda USL di Reggio

Emilia-IRCCS, Reggio Emilia, Italy

Tel +390522296073

Email Francesco.Menzella@ausl.re.it
Purpose: Severe eosinophilic asthma (SEA) is characterized by high eosinophilia, severe symptoms, important comorbidities, frequent exacerbations, and poor asthma control. Benralizumab, targeting the interleukin-5 receptor alpha, proved effective in inducing rapid eosinophil depletion and amelioration of symptoms and lung function; it also allowed to reduce exacerbations and the use of oral corticosteroids (OCS). The present case series, spanning different subtypes of SEA, aimed at expanding the real-world experience with benralizumab in Italy.

Patients and Methods: We collected data from SEA patients treated with benralizumab, at baseline and during treatment. We focused on the effects of benralizumab in the following conditions and endpoints: i) overlap between high-IgE and high-eosinophilic asthma; ii) presence of nasal polyposis as comorbidity; iii) corticosteroid-sparing effect; iv) patient perception.

Results: Ten SEA patients (females: $\mathrm{N}=7$; age range: 19-70 years) referred to 8 Italian Centers and treated with benralizumab were included, presenting with several comorbidities such as non-allergic disease (8/10), atopy (3/10), high IgE (5/10) and nasal polyposis (6/10). Overall, benralizumab yielded optimal disease control in all patients, particularly in terms of rapid clinical and functional improvement, decreased systemic steroid need (OCS therapy was completely discontinued in 7 cases) and amelioration of patient quality of life, except for 1 case, in whom other conditions not related to benralizumab therapy interfered with the patient perception.

Conclusion: Our findings further support the efficacy and safety of benralizumab observed in randomized clinical trials, providing even better results for lung function improvement.

Keywords: benralizumab, IL-5 receptor, oral corticosteroids, overlap IgE/eosinophilic asthma, polyposis, real world, severe eosinophilic asthma

\section{Introduction}

Severe asthma (SA) is a complex heterogeneous and debilitating condition associated with relevant morbidity and cost of illness. ${ }^{1}$ More than half of SA patients present a distinct phenotype characterized by high eosinophilia, severe symptoms, important comorbidities such as chronic rhinosinusitis with nasal polyposis, frequent exacerbations and poor asthma control culminating in dismal prognosis. ${ }^{2,3}$ Eosinophilic inflammation is present in approximately $50 \%$ of patients with asthma and is associated with asthma severity, higher frequency of exacerbations, and decreased lung function. ${ }^{4-6}$ Interleukin (IL)-5 is the most important factor 
influencing the differentiation, maturation and prolonged survival of eosinophils. ${ }^{7}$ In Italy, data from real-world registries indicate that SA affects approximately 200,000 people ( $5 \%$ of total asthmatics), ${ }^{1,8}$ of whom $58.8 \%$ to $71.2 \%$ present the eosinophilic phenotype (SEA). ${ }^{8,9}$

The goals of long-term asthma management are to achieve good symptom control and maintain health status, to minimize the risk of disease-related deaths, exacerbations, persistent airflow limitation and side effects and to reduce the use of oral corticosteroids (OCS), without overlooking the patient's preferences. ${ }^{10}$

Recent advances in the pathophysiology of type-2 (T2) high SA have led to the approval by the Food and Drug Administration of five monoclonal antibodies (mAb) targeting the key pathogenetic pathways that drive inflammation: omalizumab, directed against immunoglobulin E (IgE); mepolizumab and reslizumab, against IL-5; benralizumab, against the IL-5 receptor alpha (IL-5R $\alpha$ ); dupilumab, against the interleukin-4 receptor subunit $\alpha(\mathrm{IL}-4 \mathrm{R} \alpha) .{ }^{11}$ In Italy, benralizumab has been available since 2018. Due to its mechanisms of action, compared to the other mAbs, it has the advantages of inducing rapid and almost complete eosinophil depletion within 24 hours of administration (via the afucosylation-dependent Ab-dependent cell-mediated cytotoxicity) and of requiring less frequent dosing. ${ }^{7,12-14}$ Benralizumab is indicated as an add-on maintenance treatment in adult patients with SEA inadequately controlled despite high-dose inhaled corticosteroids (ICS) plus longacting $\beta$-agonists (LABA). ${ }^{15}$ In the pivotal Phase III studies SIROCCO, ${ }^{16}$ ZONDA $^{17}$ and CALIMA, ${ }^{18}$ compared to placebo, benralizumab was shown to reduce the annual rate of exacerbations by up to $70 \%$ and of hospitalization/emergency room (ER) access by up to $93 \%$, to improve symptoms and lung function (even in patients with fixed airflow obstruction) $)^{19}$ and to allow significant reduction in OCS use resulting in discontinuation in up to $52 \%$ of patients. ${ }^{17}$ This is particularly important, considering that the regular use of OCS exposes patients to an increased risk of relevant adverse outcomes (and associated costs), such as osteoporosis, osteoporotic fractures, blood hypertension, type-2 diabetes, weight gain and cataracts. ${ }^{1,8,20-22}$ Notably, the extension trial BORA $^{23}$ as well as two 2-year integrated analyses confirmed the long-term efficacy and safety of benralizumab, even in terms of maintenance of OCS dosage reduction in patients with uncontrolled SEA. ${ }^{24,25}$

As the introduction of benralizumab in clinical practice is recent, its efficacy and safety in the real-world setting are documented only by small studies (mainly retrospective), case series and case reports. Nonetheless, they corroborate the registration trials' findings, especially in terms of decreased exacerbations and OCS use. ${ }^{26-38}$ In particular, few small studies including patients with allergic EA confirmed the rapid and effective action of benralizumab already after 4 weeks in clinical practice. ${ }^{31,34,39}$

To expand the real-life experience with benralizumab in Italy, we collected data from 10 patients with SEA (8 non-allergic and 2 allergic) treated for up to 1 year. Close attention was given to the effects of benralizumab in the following conditions and endpoints i) overlap between high-IgE and high-eosinophilic asthma (as it seems to be effective regardless of serum $\mathrm{IgE}$ and atopy status), ${ }^{26,40}$ ii) presence of nasal polyposis as comorbidity, iii) corticosteroid-sparing effect and iv) perception of treated patients.

\section{Case Series}

We present data from 10 SEA patients referred to 8 Italian Centers and treated with benralizumab as per local label (30 mg subcutaneously every 4 weeks for the first three doses, and then every 8 weeks). ${ }^{15}$ Retrieved data included demographics, phenotype (allergic/nonallergic), clinical history, as well as eosinophil count, lung function and symptom control before the start of benralizumab therapy (baseline) and during treatment. Table 1 summarizes the main baseline characteristics. Figure 1 illustrates the temporal change of eosinophil count (A), Asthma Control Test (ACT) (B) and forced expiratory volume in 1 second (FEV1, expressed as percentage [\%] of predicted) (C) recorded before and during benralizumab treatment. Since patients were followed in different Centers, data collection is not fully homogeneous.

The coordinator institutional review board belongs to the Arcispedale Santa Maria Nuova, Azienda USL di Reggio Emilia-IRCCS, Reggio Emilia, Italy. All local institutional review board approved the study (Azienda USL di Reggio Emilia-IRCCS, Reggio Emilia, Italy; Ospedale GeArenzano, ASL3-Genovese, Italy; Università Cattolica del Sacro Cuore, Rome, Italy; Monaldi Hospital AO Dei ColliNaples, Italy; Ospedale Giovanni Paolo II Lamezia Terme, Italy; University of Perugia, Perugia, Italy; Santa Maria della Speranza Hospital, Battipaglia, Salerno, Italy; University of Naples Federico II, Naples, Italy; University of Salerno, Italy; University "Magna Graecia" of Catanzaro, Italy). All subjects were treated according to the standards of Good Clinical Practice and the principles of the Declaration of Helsinki and a written informed consent for publication of their details was obtained from every patients 


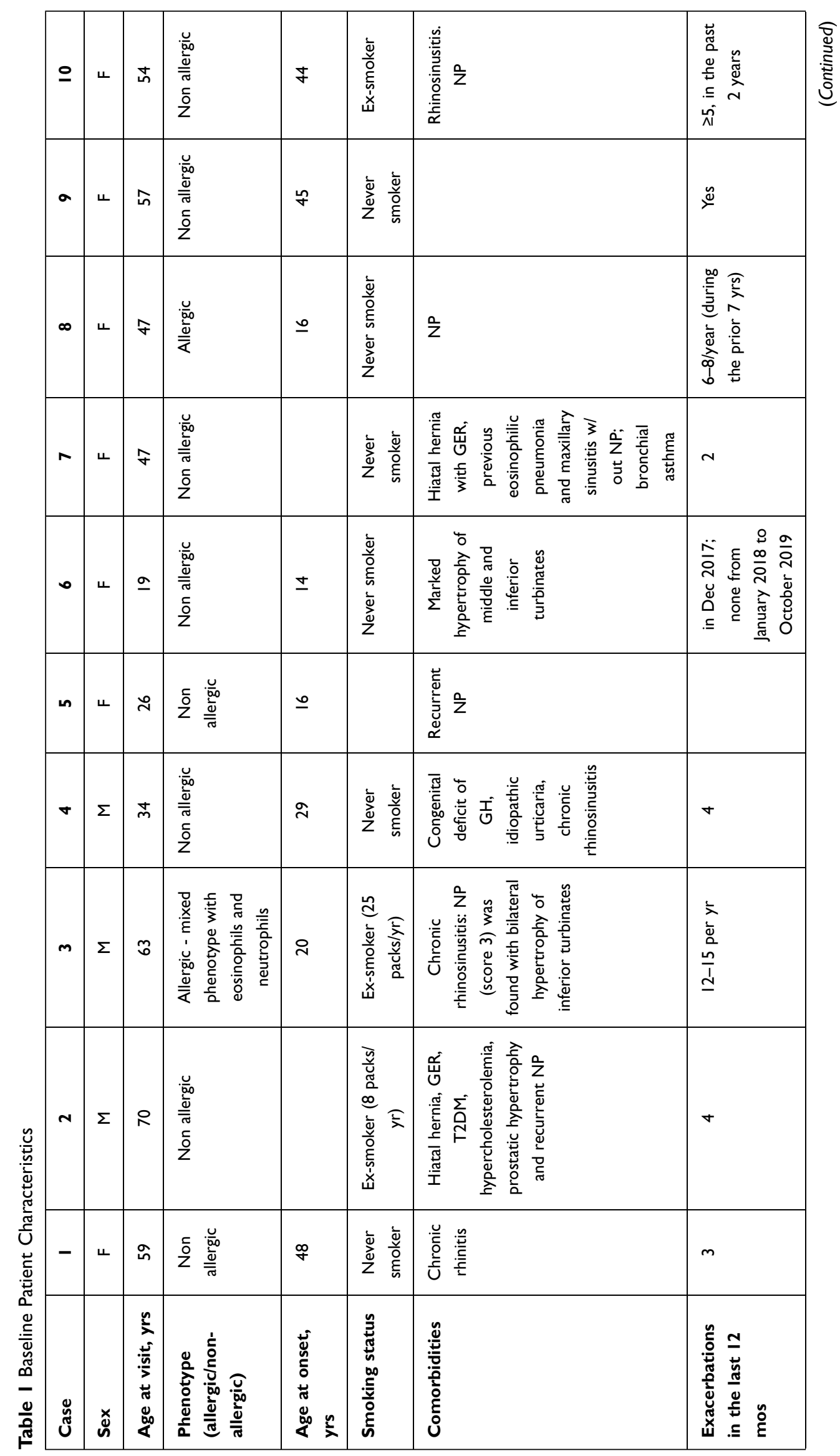




\begin{tabular}{|c|c|c|c|c|c|c|c|c|c|}
\hline 으 & 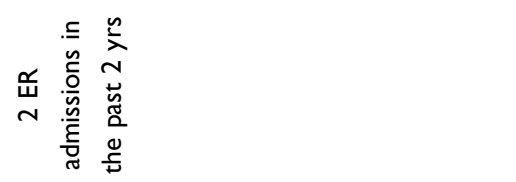 & $\stackrel{y}{\nu}$ & zo & ○ & & $\bar{N}$ & & \begin{tabular}{|l}
$\stackrel{\sim}{\tilde{I}}$ \\
$\underline{\mathbf{I}}$
\end{tabular} & 员 \\
\hline a & $\begin{array}{l}\stackrel{0}{\tilde{O}} \\
\text { z }\end{array}$ & $\stackrel{y}{\nu}$ & 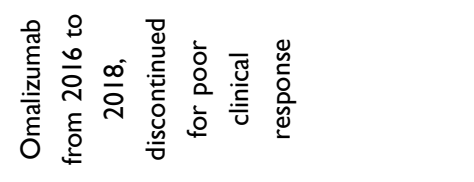 & $\hat{m}$ & & $\frac{\dot{\sigma}}{\sigma}$ & & $\underline{n}$ & in \\
\hline$\infty$ & 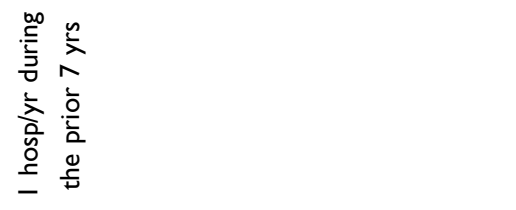 & $\stackrel{y}{\nu}$ & 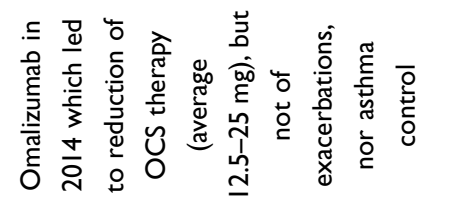 & o & & in & & $\infty$ & స్రి \\
\hline$\sim$ & $\begin{array}{l}\stackrel{0}{0} \\
\text { zo }\end{array}$ & 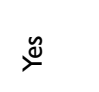 & 을 & \begin{tabular}{|l}
$\infty$ \\
$\stackrel{+}{0}$
\end{tabular} & & $\begin{array}{l}\infty \\
\end{array}$ & & 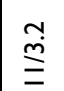 & 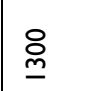 \\
\hline ○ & 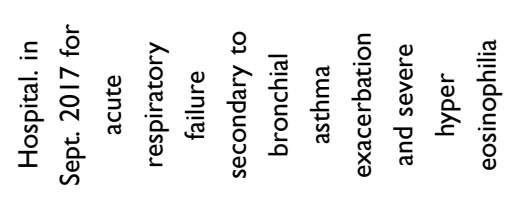 & $\stackrel{\tilde{y}}{\nu}$ & 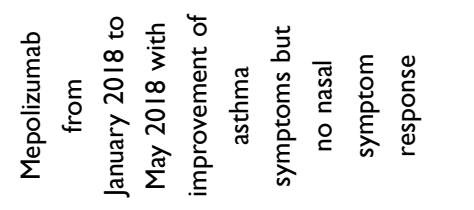 & & & & & & 周 \\
\hline in & & $\stackrel{\tilde{s}}{\nu}$ & $\stackrel{0}{z}$ & R & & & & \pm & ్ㅗ \\
\hline$\sigma$ & $\begin{array}{l}0 \\
\stackrel{0}{0}\end{array}$ & $\stackrel{y}{\nu}$ & zo & $\stackrel{n}{\sim}$ & ì & นก & $\infty$ & $\stackrel{\stackrel{\sim}{N}}{\stackrel{S}{S}}$ & 号 \\
\hline$m$ & 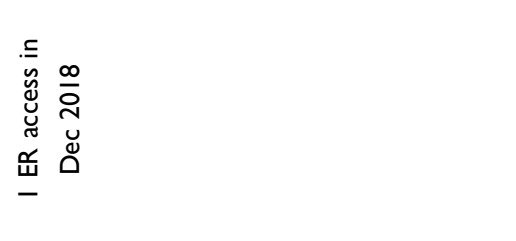 & $\stackrel{\mathscr{y}}{\tau}$ & 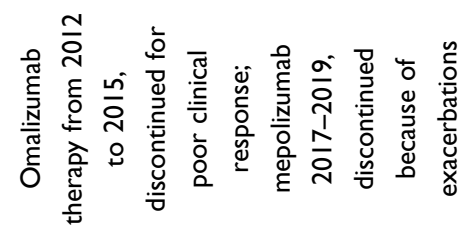 & $\stackrel{\infty}{\sim}$ & 岕 & જે & $\stackrel{\infty}{\infty}$ & \begin{tabular}{|l}
$\stackrel{\sim}{\mathfrak{N}}$ \\
$\underline{m}$
\end{tabular} & $\hat{\sigma}$ \\
\hline$N$ & & $\stackrel{\tilde{\nu}}{\check{\nu}}$ & 운 & 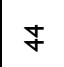 & $\frac{t}{\Lambda}$ & ๖ & $\cong$ & \pm & స్ల \\
\hline- & $\begin{array}{l}0 \\
\stackrel{0}{Z}\end{array}$ & $\stackrel{y}{x}$ & 우 & $\infty$ & & & & $\underline{\infty}$ & ○ \\
\hline נ̆ & 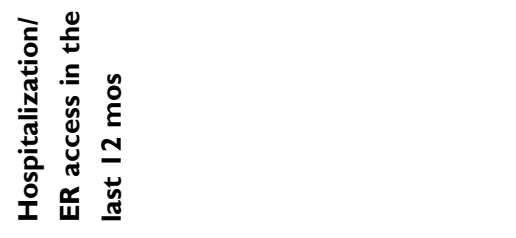 & 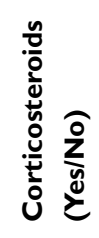 & 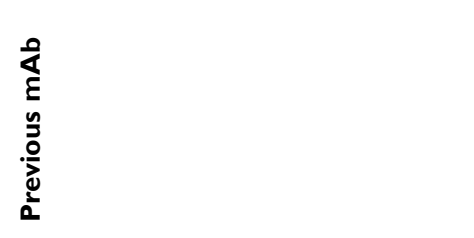 & $\stackrel{\circ}{\dot{m}}$ & 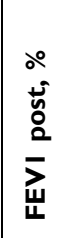 & 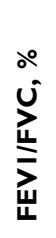 & $\begin{array}{l}2 \\
\vdots \\
0 \\
z_{0}^{0} \\
\dot{L}\end{array}$ & 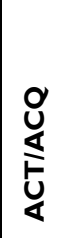 & 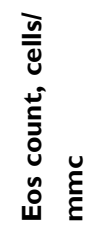 \\
\hline
\end{tabular}




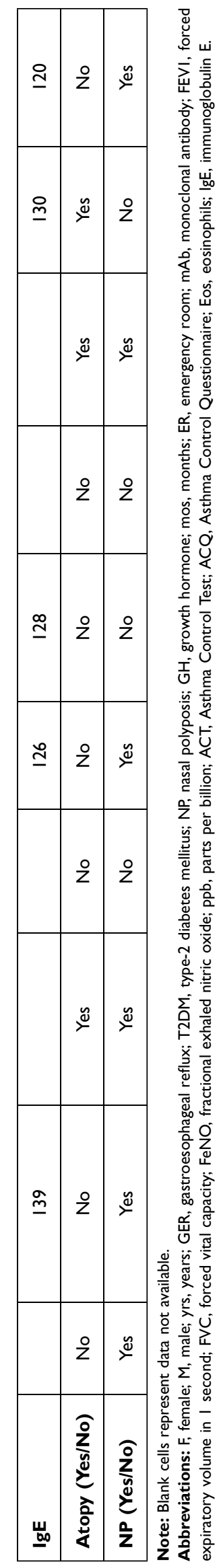

involved in this study. The authors are available to share these forms with the journal editorial office if requested.

\section{Case I}

This is the case of a 59-year-old female diagnosed with asthma at the age of 48; her mother suffered from asthma; body mass index (BMI) was 28.1; Prick test and anamnesis were negative for respiratory allergies.

At the first visit (February 2019), the patient reported asthma worsening in the previous 12 months, which had required oral cortisone every 2 to 3 months. She displayed noisy breathing during exhalation and a worsening by spirometry (FEV1: 67\% of predicted) compared to a previous test in 2017 (FEV1 87\%), together with rhinitis symptoms and chronic rhinitis. High-dose therapy with LABA and an ICS, and a short cycle with oral prednisone ( $25 \mathrm{mg}$ for 4 days, $12.5 \mathrm{mg}$ for 4 more days) were started, and a blood test was performed two weeks after OCS discontinuation. Given the blood eosinophil count of 850 cells $/ \mathrm{mmc}$ and the clinical history, the patient was prescribed benralizumab starting from the following visit.

In March 2019, the patient reported improvement soon after the start of OCS; FEV1 was $86 \%$ and ACT 18. She received the I administration of benralizumab, together with the prescription to continue high-dose inhaled therapy (vilanterol/fluticasone furoate 184 in the morning and beclomethasone/formoterol 200 in the evening). In April 2019 (1 month after the I administration of benralizumab), the patient noticed amelioration of breathing and quality of life (QoL); also lung function and symptom control improved. ICS therapy was modified following the patient's request to reduce it, ie, budesonide/formoterol fumarate dihydrate 160 (two doses) in the morning and in the evening.

Following the II administration of benralizumab, no blood eosinophils were detected. The Turbu+ diary showed that in the past 2 weeks she had autonomously decided to reduce the inhaled therapy almost to interruption. FEV1 was $86 \%$ and ACT 23 . The physician advised to restart ICS therapy and reduce it over time more carefully.

Following the III administration of benralizumab, further improvement of lung function and symptom control occurred, and olfactory function was partly regained.

After the IV administration of benralizumab, in September 2019, parameters remained quite stable; the patient received a low-dose inhaled therapy and declared to be satisfied with the result; at this time, she received the $\mathrm{V}$ administration of benralizumab. 

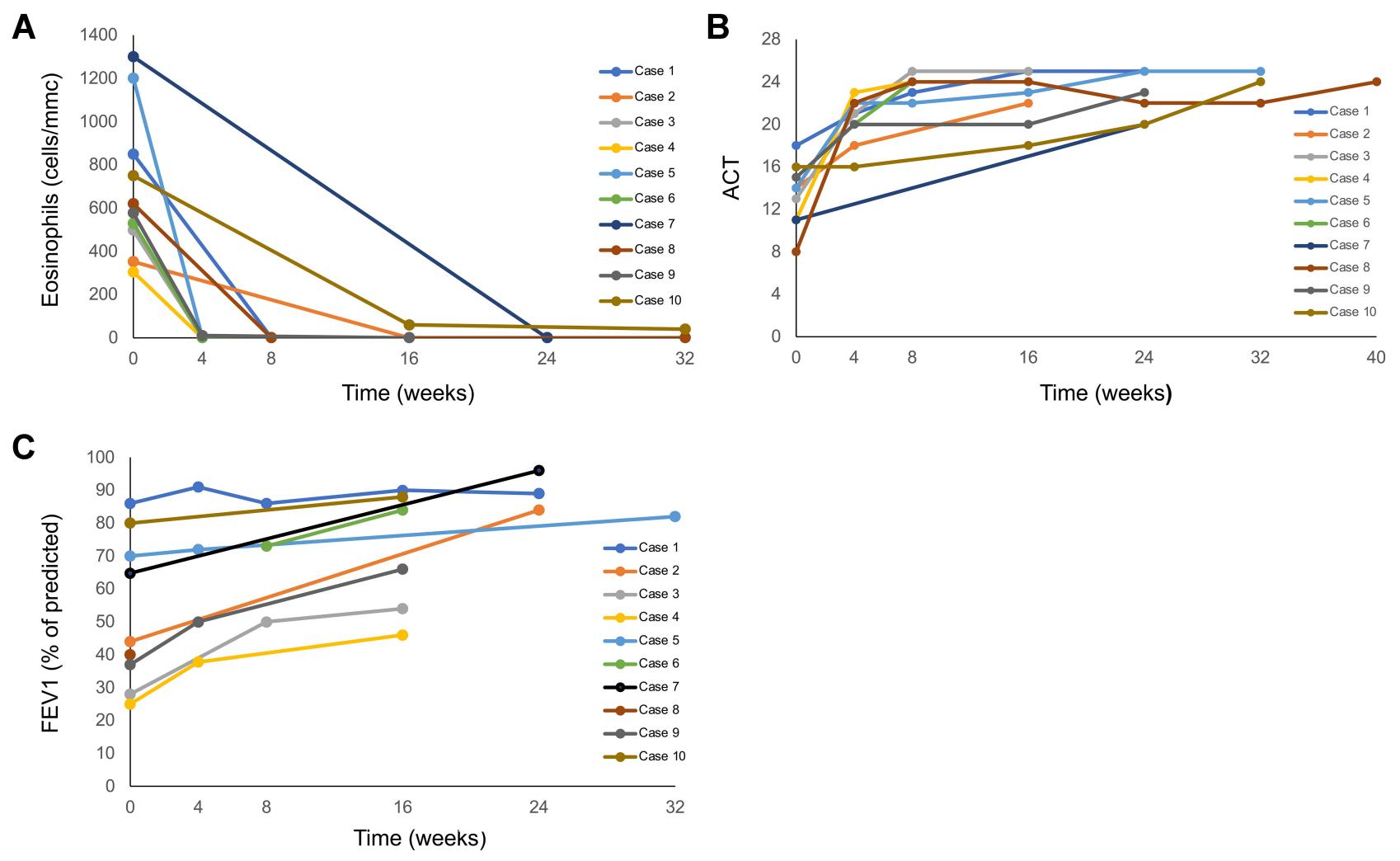

Figure I Values of eosinophil count (A), ACT (B) and FEVI (C) recorded in each patient at baseline and during benralizumab treatment.

In conclusion, the patient reported clinical and functional amelioration since the I administration of benralizumab. Notably, no OCS was necessary during the first 6 months and, after the II administration, the patient discontinued inhaled therapy for two weeks and presented good clinical-functional conditions. Since the III administration, she continued on low-dose inhaled therapy, and in the previous 2 months, the posology of budesonide/formoterol fumarate dihydrate 160 varied between 1 and 2 doses/die (she took 4 doses/die only once over two months).

\section{Case 2}

A 70-year-old Caucasian male was referred to our Asthma Centre for a worsening in respiratory symptoms after having reduced the dose of OCS upon the advice of the General Practitioner. He was a retired engineer, with a history of occupational exposure to chemical compounds and powders and an ex-smoker (smoking habit of 8 pack/year). The patient denied any alcohol or illicit drug abuse and reported productive cough, exertional dyspnea, nocturnal chest tightness, nasal obstruction and at least 4 acute exacerbation episodes over the previous year treated with high-dose OCS and antibiotics. Comorbidities included recurrent nasal polyps despite numerous surgical interventions. No allergies to inhalant allergens were reported. Daily medications included prednisone $12.5 \mathrm{mg}$, levofloxacin $500 \mathrm{mg}$, metformin, simvastatin, terazosin and high-dose ICS/LABA combinations. Tiotropium, prescribed in the past, had been stopped because of the prostatic hypertrophy.

On clinical examination, the patient appeared chronically ill, with an oxygen saturation of $96 \%$ at rest. At chest auscultation, there were bilateral basal rhonchi and diffuse wheezing. Laboratory tests showed a peripheral eosinophilic cell count of $352 / \mathrm{mmc}$ and a total serum IgE value of $139 \mathrm{IU} /$ L. Specific IgE tests (RAST) were weakly positive to grass mix. Pulmonary Function Test (PFT) revealed a severe obstructive deficit (FEV1 44\%, FEV1/forced vital capacity [FVC] $45 \%$ ) with a positive response to the reversibility test after the administration of salbutamol $400 \mathrm{mcg}$ (FEV1 post: $+14 \%$ ). The measurement of fractional exhaled nitric oxide (FeNO) was $112 \mathrm{ppb}$, markedly above the threshold clearly suggestive of uncontrolled airways inflammation (ie, 50 ppb). The ACT score of 14 supported the lack of disease control. The patient also underwent a high-resolution chest tomography (HRCT) that documented central bronchiectasis with concomitant ground-glass opacities. 
In view of the diagnosis of non-controlled SA, as recommended by international guidelines, ${ }^{10,41}$ we decided to start a biological treatment on top of maximal inhaled therapy with the aim of improving respiratory symptoms, as well as reducing exacerbation rate and systemic corticosteroid use. The patient did not fit the eligibility criteria for an anti-IgE strategy due to the lack of a sensitization to a perennial inhalant allergen. Among the anti-IL-5 options, benralizumab was chosen to provide an immediate relief to the patient and in consideration of our personal positive experience in patients with comorbidity as nasal polyps. The characteristics of the drug, including the efficacy and safety profile, were carefully explained. The ACT score, evaluated at each administration, showed a significant improvement in the degree of respiratory symptoms up to a complete asthma control (4-point increment after 8 weeks and a total score of 22 after 20 weeks). PFT and inflammatory biomarkers were re-evaluated on the IV administration of benralizumab showing an impressive improvement in FEV1 ( $>40 \%$ compared to the baseline value) and a significant reduction in FeNO values (ie, 40 ppb). Eosinophilic blood count was reduced to $0 \%$ and no further acute exacerbations were referred. Regretfully, the patient did not report an improvement in his QoL because of the occurrence of an episode of urinary tract infection (not related to benralizumab administration) which required prolonged hospitalization and led to prostatic hypertrophy worsening.

In conclusion, the addition of benralizumab provided relevant improvements in all the measured endpoints (clinical, functional and inflammatory) in a patient with highIgE/high-eosinophilic asthma. However, in the present case report, the occurrence of a concomitant health issue prevented from obtaining benefits in patient-reported outcomes, highlighting how a key-component for optimally controlling severe asthma is the proper identification and management of comorbidities. ${ }^{42}$

\section{Case 3}

This is the case of a 63-year-old male, ex-smoker (25 packs/year), with allergic bronchial asthma since the age of 20, skin sensitivity to house dust mites, grass pollen and Parietaria, and skin dander of cats and dogs.

In the past 10 years, he had suffered from chronic rhinosinusitis treated with ICS. During an Ear, Nose and Throat visit with rhino-fibroscopy 6 months earlier, nasal polyposis (score 3 ) with bilateral hypertrophy of inferior turbinates was found, with indication for surgery.
Exacerbations were 12 to 15 per year and were treated with OCS (on average $12.5 \mathrm{mg} / \mathrm{die}$ ) with an average of 20 working days lost for asthma. Blood eosinophil count was 510 cells/mmc.

He received omalizumab therapy from 2012 to 2015, which was discontinued for poor clinical response.

In November 2017, the following parameters were recorded: ACT 10; Asthma Control Questionnaire (ACQ) 22; FEV1 31\%; FVC 52\%. Mepolizumab was given for high eosinophil count $(910$ cells/mmc, with a mixed phenotype with neutrophils and eosinophils in induced sputum) until January 2019, when the therapy was discontinued because of exacerbation episodes.

In March 2019, the parameters were: blood eosinophils 497 cells/mmc; FEV1 pre 28\%; FEV1 post 34\%; FVC pre $42 \%$; FVC post 54\%; ACT 13; ACQ5 25; FeNO 88 ppb. He received inhaled budesonide/formoterol 160/4.5 2 bid, tiotropium bromide, montelukast sodium $10 \mathrm{mg}$ once/die, mometasone spray once/die, azithromycin $500 \mathrm{mg}$ thrice a week. Due to the high number of exacerbations, even infective in nature, the failure of previous biologic therapies and the severe bronchial obstruction, the decision was made to start treatment with benralizumab plus azithromycin with great symptom control.

Complete eosinophil depletion and improvement in all the clinical and functional parameters were observed already four weeks after the I administration and remained quite stable or further improved following the II and the III administration. No exacerbation occurred until February 2020. The patient reduced the OCS dose to $5 \mathrm{mg} /$ die starting from the first month of therapy, and after 3 months he stopped.

\section{Case 4}

This is the case of a 34-year-old male, never smoker, on replacement therapy for a congenital deficit of growth hormone and suffering from idiopathic urticaria and chronic rhinosinusitis without allergy. He had dyspnea since the age of 16, but the diagnosis of SA was made 5 years before the visit. No exacerbations but a severe reversible bronchial obstruction were reported.

The patient had good adherence to inhaled therapy with budesonide/formoterol 160/4.5 2 bid and tiotropium bromide; moreover, he was on chronic treatment with prednisone $10 \mathrm{mg} /$ die, with dyspnea worsening upon discontinuation.

In October 2019, the following parameters were recorded: blood eosinophils 305 cells/mmc (as measured after discontinuation of the 18-day steroid therapy); FEV1 
25\%; FVC 48\%; post FEV1 32.4\% (Rev.+13\%); ACT 11; ACQ5 20; FeNO 8; A1AT within the normal range; PRIST 4.7. The HRCT showed widespread deficit caused by abnormal perfusion bilaterally and some bronchiectasis at the right base.

Due to the young age, the severe obstruction, and the chronic therapy with OCS, benralizumab was taken into consideration. However, given the eosinophil count of 305 cells/mmc, further phenotypic assessments were carried out on the induced sputum, unveiling the presence of $8 \%$ eosinophils. One week later, the patient received the I administration of benralizumab. After one month, eosinophils were $0 \%$ in blood and $0.5 \%$ in sputum and all the parameters had improved. Prednisone dose was reduced to $2.5 \mathrm{mg}$ /die and, one month later, further to $2.5 \mathrm{mg}$ two days per week. One month before the IV administration of benralizumab, the patient discontinued the steroid therapy, and the clinical and functional improvement was still evident.

The patient declared to feel much better even if the bronchial obstruction remained important and, therefore, dyspnea had improved but was not resolved; no more exacerbations were reported.

In conclusion, this case report supports the efficacy of benralizumab in case of asthma and fixed obstruction.

\section{Case 5}

This is the case of a 26-year-old female diagnosed with asthma at the age of 16 . Nasal polyposis had been surgically treated eight years before but had occurred again in the past two years, and, at the time of the visit, was treated with ICS; she reported anosmia. Tests for common inhalant allergens were negative. The patient displayed sour breath with diffuse obstruction.

Blood eosinophil count was 1200/mmc; total IgE 126 IU/L; FEV1 by spirometry 70\%; ACT 14 . She was on treatment with ICS/LABA, combined with LAMA since few months, and had received daily OCS (prednisone $25 \mathrm{mg} / \mathrm{die}$ ) in the past 3 months.

In October 2018, benralizumab therapy was started. On day 28, no blood eosinophils were detected, IgE value was $38 \mathrm{IU} / \mathrm{L}$ and both lung function and symptom control were improved; notably, the use of OCS had been reduced. At the last follow-up, in July 2019, blood eosinophils were still undetectable, IgE were $26 \mathrm{IU} / \mathrm{L}$ and a further clinical and functional improvement was observed. OCS therapy had been discontinued three months before. The patient did not experience any exacerbation, hospitalization or ER access throughout benralizumab therapy.

In conclusion, in this case with high eosinophils/high IgE, benralizumab yielded a rapid and sustained improvement of all clinical and functional parameters, allowing to discontinue OCS therapy.

\section{Case 6}

This is the case of a 19-year-old female diagnosed with non-allergic bronchial EA in 2014, presenting with perennial rhinitis since September 2014 with marked hypertrophy of middle and inferior turbinates associated with anosmia and ageusia; skin allergy tests were negative; dosing of IgE specific for inhaled allergens was negative. An intervention of septoplasty with turbinoplasty in February 2018 required corticosteroid therapy for $>6$ months/year (average dose of prednisone: $12.5 \mathrm{mg} / \mathrm{die}$ ) and frequent cycles of antibiotics; approximately 60 schooldays were lost in the previous year.

In September 2016, the HRCT was normal; in September 2017, she was hospitalized in Pneumology Department for acute respiratory failure secondary to bronchial asthma exacerbation and severe hypereosinophilia; in November 2017, ACT was 18; ACQ 3.6; AQLQ 4.03. In December 2017, SA exacerbation occurred, requiring oral steroids (prednisone $50 \mathrm{mg} / \mathrm{die}$, then tapering). She received mepolizumab from January 2018 to May 2019 with the improvement of asthma symptoms but no nasal symptom response. In September 2018, FEV1 was 95\%. Ongoing therapy was as follows: beclomethasone/formoterol 200/6 mcg spray twice a day; montelukast biomarkers were: blood eosinophils were 530 cells $/ \mathrm{mmc}$, total $\mathrm{IgE} 128 \mathrm{IU} / \mathrm{L}$ and FeNO 77 ppb.

In July 2019, she started benralizumab therapy with improvement in nasal symptoms and complete asthma control. No new exacerbation was observed from January 2018 to October 2019. In September 2019, OCS therapy was discontinued, and the blood biomarkers were markedly improved: eosinophils 0 cells/mmc; total $\mathrm{IgE} 35$ $\mathrm{UI} / \mathrm{L}$; FeNO 12 ppb. Asthma and QoL amelioration were confirmed by questionnaires in October 2019.

In conclusion, in this case of high-IgE/higheosinophilic asthma, mepolizumab provided a good control of asthma but not of nasal symptoms. After switching to benralizumab, asthma control persisted, and a rapid and relevant improvement of nasal symptoms occurred, with reduction of obstruction and partial gain in smell and taste. Notably, OCS therapy was discontinued. 


\section{Case 7}

A 47-year-old woman was referred to our Severe Asthma Center in April 2017 to possibly undergo bronchial thermoplasty. She was a never-smoker administrative employee with a clinical history of hiatal hernia with gastroesophageal reflux, previous eosinophilic pneumonia, and maxillary sinusitis without nasal polyposis. No sensitization to seasonal and perennial inhalant allergens was reported. Bronchial asthma started in 2003 with progressive worsening of control and 3 severe exacerbations in the last 12 months. Her current medications included pMDI beclomethasone/formoterol 200/6 mcg twice a day, tiotropium bromide $2.5 \mathrm{mcg}$ twice a day, prednisone tablets $12.5 \mathrm{mg} /$ day, esomeprazole tablets $40 \mathrm{mg} /$ day. Spirometry showed a moderate airflow obstruction (FEV1 65\%; FEV1/FVC 0.51) without bronchodilator reversibility. At baseline, the ACT score was 11 points and the ACQ score 3.2, suggestive of uncontrolled disease. Blood eosinophils were 1300 cells $/ \mathrm{mmc}$ while the ANCA test was negative. As there was no indication for omalizumab, the patient underwent three bronchial thermoplasty standard treatment sessions at 3-week intervals between May and June 2017. In the following months, asthma control did not improve, and no reduction in the exacerbation rate, symptoms, ACT score and OCS dosage was observed.

In March 2019, the patient was re-evaluated and, in light of the presence of elevated blood eosinophilia and of the lack of response to bronchial thermoplasty, the decision was made to start benralizumab treatment. The clinical picture progressively improved and the spirometry performed after 16 weeks showed a marked increase in the parameters. After 6 months, complete blood eosinophil depletion was observed, together with a significant increase in asthma control. Four weeks after the start of treatment, OCS therapy began to be tapered and was completely withdrawn later on.

Indeed, benralizumab led to a progressive improvement in lung function and asthma control, in a patient in which bronchial thermoplasty was not sufficient to guarantee the improvement of asthma with the reduction in the exacerbation rate, symptoms, ACT score and OCS dosage.

\section{Case 8}

A 47-year-old female diagnosed with allergic bronchial asthma at 16 years of age in treatment with ICS/LABA, with skin prick test (SPT) positive for house dust mites reported progressive asthma worsening in the last 10 years after the diagnosis of nasal polyps. She was a non-smoker employed in a small enterprise, living in an urban area. Nasal polyps were treated with nasal steroids, OCS and surgery (two times) with little improvement in nasal and asthmatic symptoms. During the last 7 years, the patient experienced frequent asthma exacerbations (6-8/year), some requiring hospitalization (about 1/year) despite continuous OCS treatment (prednisone $12.5-37.5 \mathrm{mg} /$ day) and the introduction of anti-IgE monoclonal antibodies (omalizumab $300 \mathrm{mg} / \mathrm{month}$ ) in 2014. This led to OCS therapy reduction (average 12.5-25 mg) but did not improve the exacerbation rate and asthma control (average ACT 8-12). The patient experienced several OCS-related side effects, including sleep deprivation and increase in body weight and ocular pressure, but not cortisol suppression. Spirometry showed a persistent airway obstruction (reduced FEV1/FVC ratio with average FEV1 40\%) but still reversible after beta- 2 agonist administration. FeNO was largely influenced by ICS and OCS. Meanwhile, nasal symptoms worsened in the last 2 years, affecting sleep, work and physical activity, and requiring new surgery.

For this reason, given the number of blood eosinophils of 620 cells $/ \mathrm{mmc}$ and despite OCS treatment, in December 2018 the patient started benralizumab $30 \mathrm{mg} /$ month for the first 3 months and then every 2 months with a significant reduction in asthma exacerbation rate (1/year, mild), asthma symptoms and OCS use. After 6 months and adequate scaling, the patient discontinued OCS treatment, continuing ICS/LABA at a low dosage (formoterol/beclomethasone 6/100 bid). No further nasal surgery was needed, and the patient reported a significant improvement in global QoL, including work and physical activity, lung function and asthma control. No side effects were reported, and OCS discontinuation was not followed by any effect on cortisol level or symptoms. After one year of treatment, QoL was significantly improved and expenses for exacerbations, hospitalization and nasal surgery were avoided. Moreover, in the last 3 months, the patient reported a significant reduction in the airway (upper and lower) infection rate that was rather common before the treatment with benralizumab. No antibiotic therapy was necessary in the past year.

In conclusion, in a patient with SEA and nasal polyps benralizumab improved asthma control, lung function and QoL, in particular reducing work disability.

\section{Case 9}

This is the case of a 57-year-old non-smoker female. The first assessment of SA was done in June 2018, and 
uncontrolled SA with high IgE and high eosinophils was diagnosed. The onset of asthmatic symptoms had occurred 12 years before; the SPT and RAST test for inhalant allergens was negative. From 2016 to 2018 she received omalizumab therapy, but it was discontinued for poor clinical response. The following therapies were ongoing: medium-high dose of ICS/LABA plus LAMA, LTRA + aerosol therapy and SABA as needed.

In the previous 12 months, she had experienced exacerbations treated with OCS betamethasone $1 \mathrm{mg}$ as needed, $7 / 8$ times per month, without the need for hospitalization or ER or intensive care unit access; in the same period, she had undergone 6 specialized visits. No comorbidities were reported.

In December 2018, before she started benralizumab therapy, the eosinophil count was 577 cells/mmc, FEV1 $37 \%$ and ACT 15. Subsequent administrations were given in January 2019 (II), February 2019 (III), March 2019 (IV) and May 2019 (V). Eosinophil count, lung function and symptom control improved already after the I administration and the improvement persisted throughout the treatment period. No exacerbations occurred and no OCS therapy was used.

In conclusion, in this case of uncontrolled SA with high-IgE/high-eosinophilic asthma and exacerbations, benralizumab led to an overtime improvement iin eosinophil count, lung function and symptom control without OCS need.

\section{Case 10}

A 54-year-old female was evaluated in the outpatient clinic for uncontrolled SA. She worked as secretary in a lawyer study; she was a former smoker and had quit about 10 years before the visit. Asthma symptoms had started $>10$ years before, including initial main symptoms of acute episodes of dyspnea and cough occurring every 2 to 3 months and controlled by short-term course of ICS/ LABA. Approximately 8 years before the visit, the lung function test had shown moderate obstruction, partially reversible with salbutamol. The patient also referred symptoms of rhinosinusitis with nasal obstruction, frontal headache, and nasal mucosal discharge. Approximately 5 years before the first visit, the patient had undergone nasal polypectomy. In the past 2 years, at least 5 asthma exacerbations had occurred, requiring in two cases admission to the ER.

At the time of the first visit, the patient had dyspnea and cough, nasal obstruction, anosmia, frequent headache, and chronic rhinosinusitis. On physical examination, she had diffuse wheezing and pain on the paranasal sinuses. Her BMI was 27. Ongoing therapy included oral prednisone ( $5 \mathrm{mg} /$ day), fluticasone/formoterol 500/20, montelukast $(10 \mathrm{mg} /$ day) and nasal azelastine/fluticasone.

Lung function tests showed: FEV1 80\%; FEV1/FVC $71 \%$; forced expiratory flow $25-75,53 \%$. Blood eosinophils (before starting oral prednisone) were 750 cells $/ \mathrm{mmc}$ and total serum IgE $120 \mathrm{IU} / \mathrm{L}$; the Prick test for aeroallergens was negative; ACT was 16; ACQ-6 was 2.25; SNOT22 was 58 . A diagnosis of SA was made and benralizumab was started. Before the II administration, prednisone was reduced to $2.5 \mathrm{mg}$ /day for 2 weeks and then discontinued; however, the patient experienced another exacerbation requiring restarting of oral prednisone $(5 \mathrm{mg} /$ day for 5 days followed by $2.5 \mathrm{mg} /$ day). The ACT score was unchanged. Two weeks after the III injection of benralizumab, symptoms improved, and the patient reported no more dyspnea and cough, and amelioration of the nasal discharge; FEV1 increased and the count of blood eosinophils dropped; notably, the improvement persisted throughout the treatment period. Prednisone was reduced to $2.5 \mathrm{mg}$ every other day and was discontinued after 2 months, with the improvement of ACT after steroid withdrawal (ACT 20). Adherence to therapy and the correct use of the fluticasone/formoterol inhaler were reevaluated. No additional worsening of asthma was observed.

This case report underlines the difficulties in reducing OCS in patients with SA. Initiation of treatment with benralizumab allowed OCS discontinuation but the rate for steroid reduction should be tailored for each patient according to the results of the lung function test and ACT score.

\section{Discussion}

Our findings provide additional evidence of the effectiveness of benralizumab in achieving optimal disease control in patients with SEA, particularly in terms of rapid clinical and functional improvement, decreased systemic steroid need and amelioration of patient QoL.

The patients described here are representative of complex cases that physicians may encounter in daily practice outside of the controlled setting of randomized trials. None of the subjects enrolled had immune defects or cystic fibrosis. These cases were selected based on the following factors that the authors deemed as important in the management of SEA patients: i) Overlap between high-IgE and high-eosinophilic asthma: our results from 5 cases confirm 
previous data demonstrating the effectiveness of benralizumab treatment regardless of serum $\operatorname{IgE}$ and atopy status. ${ }^{26,40}$ ii) Presence of nasal polyposis as comorbidity: in our case series, it was reported in 6 cases with further improvement in all cases, in line with previous findings. ${ }^{30,31}$ Notably, nasal polyposis, which is one of the most frequent comorbidity observed in SEA patients, has been recently included among the predictors of response to benralizumab therapy. ${ }^{36,43,44}$ Indeed, nasal polyposis has been associated with higher eosinophil count and OCS use, and worse lung function, which, together, allow a better asthma control (ie, exacerbation rate), corticosteroid-sparing effect and lung function improvement upon benralizumab. iii) Corticosteroidsparing effect: ${ }^{45}$ In the present case series, the dose of steroids was tapered/discontinued in 8 cases. This is important as, according to the SANI registry, $64.1 \%$ of patients use OCS chronically, being exposed to a high risk of adverse events and related costs. ${ }^{1,8,20}$ Additionally, a recent study reported that $76 \%$ of primary care patients received at least an OCS prescription within the first year of SA diagnosis to control symptoms. ${ }^{46}$ However, these drugs have to be prescribed after appropriate assessment of factors potentially contributing to the exacerbations, and treatment optimization. ${ }^{46}$ Recently, Canonica and coworkers estimated an annual cost per patient for OCS-related comorbid conditions equal to $€ 1957,50$ (vs $€ 1350,96$ in those with mild/moderate asthma and $€ 1065,27$ in nonasthma controls), with a total annual cost of $€ 242.7$ million (vs $€ 167.5$ and $€ 132.0$, respectively). ${ }^{1}$ Notably, preliminary data from a cost-effectiveness analysis of benralizumab conducted in Sweden have shown that, compared with the standard of care plus OCS, this therapy has a high likelihood of being cost-effective for SEA patients on regular OCS treatment. ${ }^{47}$ However, more data are needed to draw definitive conclusions. iv) Perception of treated patients: Albeit in most cases patients reported amelioration of their QoL following improvement in asthma and nasal symptoms, other conditions occasionally interfered with the patient perception of his/her QoL (ie, case 2). As comorbidities are frequent in the setting of SEA, their proper identification and management are a key component to optimally control disease. ${ }^{42}$ Whether the treatment with benralizumab may represent a valuable tool for managing bronchiectasis is a matter deserving further and ad-hoc evaluation, particularly considering the high frequency of such comorbidity in patients with SA (up to $40 \%)^{48}$
In our cohort, the use of benralizumab was associated also with a marked reduction in the number of exacerbations and/or hospitalizations. A preliminary retrospective analysis of 43 SEA patients treated with benralizumab for at least 12 months demonstrated a $75 \%$ reduction in exacerbations, a $100 \%$ reduction in maintenance OCS use at 1 year, with $53.6 \%$ of patients able to entirely discontinue OCS by 1 year. ${ }^{49}$ Another analysis conducted in a realworld tertiary asthma center setting in 42 patients showed a $70 \%$ reduction in maintenance OCS dose already by week 16: despite this reduction, $79 \%$ of subjects remained exacerbation-free at week $24 .{ }^{26} \mathrm{~A}$ retrospective analysis of 9 patients with SEA treated with benralizumab for 48 weeks reported a $70.6 \%$ decrease in exacerbations at 48 weeks, which increased to $83.3 \%$ in responders, defined by complete control or marked improvement in asthma symptoms based on Global Evaluation of Treatment Effectiveness scores. ${ }^{28}$ Recently, benralizumab administered in a patient with severe asthma and eosinophilic chronic rhinosinusitis decreased the eosinophil count, improved lung function, and ameliorated eosinophilic chronic rhinosinusitis. ${ }^{29}$ Finally, recent Italian reports of patients with allergic SEA with or without nasal polyps support the rapid and sustained (ie, up to 24 weeks) effect of benralizumab in a real-world setting, especially on lung function improvement and exacerbation rate decrease. ${ }^{30,31,35}$

The main limitations of the present case series are due to the study design and to the fact that, as different centers were involved, data collection was performed as per local practice and thus is not completely homogeneous. The main strengths are represented by the relatively long follow-up, the thorough qualitative analysis, and the different subtypes of SEA presented: 8 non-allergic disease, 3 atopic; 5 with overlap between high IgE and high eosinophil count; 6 with polyposis. Although a number of real-world studies have been published with a larger sample size, the novelty of our case series resides in the fact that we detailed the evolution of each single patient on benralizumab, rather than presenting the pooled data, and in the high heterogeneity of the characteristics of the cases presented.

\section{Conclusion}

Following the evolution of each single patient has allowed to confirm previous data on the effectiveness and safety of benralizumab, providing even better results, for example, in terms of lung function improvement. This is very important, considering the real-world setting in which our patients were treated. 
A number of ongoing trials will shed more light on the use of benralizumab in SEA and other eosinophilic diseases, especially with regard to patient-reported outcomes and healthcare resource utilization.

\section{Acknowledgments}

Editorial and writing assistance was provided by Clara Ricci, PhD (Edra S.p.A., Milan, Italy) and funded by AstraZeneca.

\section{Funding}

AstraZeneca provided financial support for medical writing assistance. The funder was not involved in the collection, analysis, interpretation of data, the writing of this article or the decision to submit it for publication.

\section{Disclosure}

FM declares research fundings as Principal investigator by AstraZeneca, Chiesi Farmaceutici, Novartis, Sanofi; fees as speaker/lecturer by AstraZeneca, Chiesi Farmaceutici, GlaxoSmithKline, Novartis, Sanofi; MBonavia declares fee as a speaker from GlaxoSmithKline; MBonini has nothing to declare; MDA has nothing to declare; SL has received fees from AstraZeneca, Menarini, Glaxo, Novartis, Chiesi; NM declares non-financialsupport from GlaxoSmithKline, AstraZeneca, Chiesi, ALK-ABELLO and Menarini; VP has nothing to declare; MT declares Speaker and Advisory board fee from Novartis, AstraZeneca, Sanofi;GP has received lecture fees and consultancy fees from Alfasigma, AstraZeneca, Chiesi, GlaxoSmithKline, Guidotti-Malesci, Menarini, Mundipharma, Novartis, Sanofi, Zambon. The authors report no other conflicts of interest in this work.

\section{References}

1. Canonica GW, Colombo GL, Bruno GM, et al. Shadow cost of oral corticosteroids-related adverse events: a pharmacoeconomic evaluation applied to real-life data from the Severe Asthma Network in Italy (SANI) registry. World Allergy Organ J. 2019;12(1):100007. doi:10.1016/j.waojou.2018.12.001

2. PubMed - NCBI. Heterogeneity of phenotypes in severe asthmatics. The Belgian Severe Asthma Registry (BSAR). Available from: https:// www.ncbi.nlm.nih.gov/pubmed/25456708. Accessed December 9, 2019.

3. Price DB, Rigazio A, Campbell JD, et al. Blood eosinophil count and prospective annual asthma disease burden: a UK cohort study. Lancet Respir Med. 2015;3(11):849-858. doi:10.1016/S2213-2600(15)003 67-7

4. Zhang JY, Wenzel SE. Tissue and BAL based biomarkers in asthma. Immunol Allergy Clin North Am. 2007;27(4):623-632;vi. doi:10.1016/ j.iac.2007.09.003
5. PubMed - NCBI. Predicting frequent asthma exacerbations using blood eosinophil count and other patient data routinely available in clinical practice. Available from: https://www.ncbi.nlm.nih.gov/ pubmed/26793004. Accessed May 18, 2020.

6. Talini D, Novelli F, Bacci E, et al. Sputum eosinophilia is a determinant of FEV1 decline in occupational asthma: results of an observational study. BMJ Open. 2015;5(1):e005748. doi:10.1136/ bmjopen-2014-005748

7. Matucci A, Maggi E, Vultaggio A. Eosinophils, the IL-5/IL-5R $\alpha$ axis, and the biologic effects of benralizumab in severe asthma. Respir Med. 2019;160:105819. doi:10.1016/j.rmed.2019.105819

8. Heffler E, Blasi F, Latorre M, et al. The severe asthma network in Italy: findings and perspectives. $J$ Allergy Clin Immunol Pract. 2019;7(5):1462-1468. doi:10.1016/j.jaip.2018.10.016

9. Maio S, Baldacci S, Bresciani M, et al. RItA: the Italian severe/uncontrolled asthma registry. Allergy. 2018;73(3):683-695. doi:10.1111/ all.13342

10. 2019 GINA Main Report. Global initiative for asthma - GINA. Available from: https://ginasthma.org/gina-reports/. Accessed March 27, 2020.

11. Agache I, Beltran J, Akdis C, et al. Efficacy and safety of treatment with biologicals (benralizumab, dupilumab, mepolizumab, omalizumab and reslizumab) for severe eosinophilic asthma. Allergy. 2020. doi:10.1111/all.14221

12. PubMed - NCBI. Benralizumab, an add-on treatment for severe eosinophilic asthma: evaluation of exacerbations, emergency department visits, lung function, and oral. Available from: https:/www. ncbi.nlm.nih.gov/pubmed/30425502. Accessed December 9, 2019.

13. Pelaia C, Calabrese C, Vatrella A, et al. Benralizumab: from the basic mechanism of action to the potential use in the biological therapy of severe eosinophilic asthma. Biomed Res Int. 2018;2018:4839230. doi: $10.1155 / 2018 / 4839230$

14. Sehmi R, Lim HF, Mukherjee M, et al. Benralizumab attenuates airway eosinophilia in prednisone-dependent asthma. J Allergy Clin Immunol. 2018;141(4):1529-1532.e8. doi:10.1016/j.jaci.2018.01.008

15. fasenra-epar-product-information_en.pdf. Available from: https:// www.ema.europa.eu/en/documents/product-information/fasenra-eparproduct-information_en.pdf. Accessed December 6, 2019.

16. Bleecker ER, FitzGerald JM, Chanez P, et al. Efficacy and safety of benralizumab for patients with severe asthma uncontrolled with highdosage inhaled corticosteroids and long-acting $\beta 2$-agonists (SIROCCO): a randomised, multicentre, placebo-controlled Phase 3 trial. Lancet. 2016;388(10056):2115-2127. doi:10.1016/S0140-6736(16)31324-1

17. Nair P, Wenzel S, Rabe KF, et al. Oral glucocorticoid-sparing effect of benralizumab in severe asthma. $N$ Engl J Med. 2017;376 (25):2448-2458. doi:10.1056/NEJMoa1703501

18. FitzGerald JM, Bleecker ER, Nair P, et al. Benralizumab, an antiinterleukin-5 receptor $\alpha$ monoclonal antibody, as add-on treatment for patients with severe, uncontrolled, eosinophilic asthma (CALIMA): a randomised, double-blind, placebo-controlled phase 3 trial. Lancet. 2016;388(10056):2128-2141. doi:10.1016/S0140-6736(16)31322-8

19. Chipps BE, Hirsch I, Trudo F, Alacqua M, Zangrilli JG. Benralizumab efficacy for patients with fixed airflow obstruction and severe, uncontrolled eosinophilic asthma. Ann Allergy Asthma Immunol. 2020;124(1):79-86. doi:10.1016/j.anai.2019.10.006

20. Sweeney J, Patterson CC, Menzies-Gow A, et al. Comorbidity in severe asthma requiring systemic corticosteroid therapy: cross-sectional data from the optimum patient care research database and the British thoracic difficult asthma registry. Thorax. 2016;71 (4):339-346. doi:10.1136/thoraxjnl-2015-207630

21. Sullivan PW, Ghushchyan VH, Globe G, Schatz M. Oral corticosteroid exposure and adverse effects in asthmatic patients. J Allergy Clin Immunol. 2018;141(1):110-116.e7. doi:10.1016/j.jaci.2017.04.009

22. Price DB, Trudo F, Voorham J, et al. Adverse outcomes from initiation of systemic corticosteroids for asthma: long-term observational study. J Asthma Allergy. 2018;11:193-204. doi:10.2147/JAA.S176 026 
23. Busse WW, Bleecker ER, FitzGerald JM, et al. Long-term safety and efficacy of benralizumab in patients with severe, uncontrolled asthma: 1-year results from the BORA phase 3 extension trial. Lancet Respir Med. 2019;7(1):46-59. doi:10.1016/S2213-2600(18)30406-5

24. FitzGerald JM, Bleecker ER, Bourdin A, et al. Two-year integrated efficacy and safety analysis of benralizumab in severe asthma. J Asthma Allergy. 2019;12:401-413. doi:10.2147/JAA.S227170

25. Bourdin A, Shaw D, Menzies-Gow A, et al. Two-year integrated steroid-sparing analysis and safety of benralizumab for severe asthma. J Asthma. 2019;1-9. Doi:10.1080/02770903.2019.1705333.

26. Jackson D, Roxas C, Thompson L, et al. Real-world oral glucocorticoid-sparing effect of benralizumab in severe asthma. Eur Respir J. 2019;54(suppl 63). doi:10.1183/13993003.congress-2019. PA2527

27. Kavanagh JE, Hearn AP, Dhariwal J, et al. Real-world effectiveness of benralizumab in severe eosinophilic asthma. Chest. 2020;158:491-500. doi:10.1016/j.chest.2020.08.2083

28. Murphy E, Diaz J, Singh H. P215 real life experience with benralizumab in patients with severe uncontrolled eosinophilic asthma. Ann Allerg Asthma Immunol. 2019;123(5):S36-S37. doi:10.1016/j.anai.2019.08.281

29. Tsurumaki H, Matsuyama T, Ezawa K, et al. Rapid effect of benralizumab for hypereosinophilia in a case of severe asthma with eosinophilic chronic rhinosinusitis. Medicina (Kaunas). 2019;55(7). doi:10.3390/medicina55070336

30. Pelaia C, Busceti MT, Vatrella A, et al. Effects of the first three doses of benralizumab on symptom control, lung function, blood eosinophils, oral corticosteroid intake, and nasal polyps in a patient with severe allergic asthma. SAGE Open Med Case Rep. 2020; 8:2050313X20906963. doi:10.1177/2050313X20906963

31. Pelaia C, Busceti MT, Vatrella A, et al. Real-life rapidity of benralizumab effects in patients with severe allergic eosinophilic asthma: assessment of blood eosinophils, symptom control, lung function and oral corticosteroid intake after the first drug dose. Pulm Pharmacol Ther. 2019;58:101830. doi:10.1016/j.pupt.2019.101830

32. Voelker D, Almodallal Y, Scrodin MD, et al. Newer biological agents in the treatment of severe asthma: real-world results from a tertiary referral center. Lung. 2020;198:653-659. doi:10.1007/s00408-020-00369-8

33. Kotisalmi E, Hakulinen A, Mäkelä M, Toppila-Salmi S, Kauppi P. A comparison of biologicals in the treatment of adults with severe asthma - real-life experiences. Asthma Res Pract. 2020;6:2. doi:10.1186/s40733-020-00055-9

34. Izumo T, Tone M, Kuse N, et al. Effectiveness and safety of benralizumab for severe asthma in clinical practice (J-BEST): a prospective study. Ann Transl Med. 2020;8(7):438. doi:10.21037/atm.2020.04.01

35. Pelaia C, Busceti MT, Crimi C, et al. Real-Life effects of benralizumab on exacerbation number and lung hyperinflation in atopic patients with severe eosinophilic asthma. Biomed Pharmacother. 2020;129:110444. doi:10.1016/j.biopha.2020.110444

36. Menzella F, Ruggiero P, Galeone C, Scelfo C, Bagnasco D, Facciolongo N. Significant improvement in lung function and asthma control after benralizumab treatment for severe refractory eosinophilic asthma. Pulm Pharmacol Ther. 2020;64:101966. doi:10.1016/j. pupt.2020.101966
37. Di Bona D, Minenna E, Albanesi M, Nettis E, Caiaffa MF, Macchia L. Benralizumab improves patient reported outcomes and functional parameters in difficult-to-treat patients with severe asthma: data from a real-life cohort. Pulm Pharmacol Ther. 2020;64:101974. doi:10.1016/j.pupt.2020.101974

38. Bagnasco D, Brussino L, Bonavia M, et al. Efficacy of Benralizumab in severe asthma in real life and focus on nasal polyposis. Respir Med. 2020;171:106080. doi:10.1016/j.rmed.2020.106080

39. Bergantini L, d'Alessandro M, Cameli P, et al. Personalized approach of severe eosinophilic asthma patients treated with mepolizumab and benralizumab. Int Arch Allergy Immunol. 2020;181(10):746-753. doi:10.1159/000508936

40. Chipps BE, Newbold P, Hirsch I, Trudo F, Goldman M. Benralizumab efficacy by atopy status and serum immunoglobulin E for patients with severe, uncontrolled asthma. Ann Allergy Asthma Immunol. 2018;120(5):504-511.e4. doi:10.1016/j.anai.2018.01.030

41. Holguin F, Cardet JC, Chung KF, et al. Management of severe asthma: a European Respiratory Society/American Thoracic Society guideline. Eur Respir J. 2020;55(1):1900588. doi:10.1183/139930 03.00588-2019

42. Gibson PG, McDonald VM. Management of severe asthma: targeting the airways, comorbidities and risk factors. Intern Med J. 2017;47 (6):623-631. doi:10.1111/imj.13441

43. Bleecker ER, Wechsler ME, FitzGerald JM, et al. Baseline patient factors impact on the clinical efficacy of benralizumab for severe asthma. Eur Respir J. 2018;52(4):1800936. doi:10.1183/13993003. 00936-2018

44. FitzGerald JM, Bleecker ER, Menzies-Gow A, et al. Predictors of enhanced response with benralizumab for patients with severe asthma: pooled analysis of the SIROCCO and CALIMA studies. Lancet Respir Med. 2018;6(1):51-64. doi:10.1016/S2213-2600(17) 30344-2

45. Menzella F, Biava M, Bagnasco D, et al. Efficacy and steroid-sparing effect of benralizumab: has it an advantage over its competitors? Drugs Context. 2019;8:212580. doi:10.7573/dic.212580

46. Vetrano DL, Zucchelli A, Bianchini E, et al. Patterns of oral corticosteroids use in primary care patients with severe asthma. Respir Med. 2020;166:105946. doi:10.1016/j.rmed.2020.105946

47. Andersson M, Janson C, Kristensen T, Szende A, Golam S. PRS24 cost effectiveness of benralizumab for severe, uncontrolled oral corticosteroid-dependent asthma in Sweden. Value Health. 2019;22: S876-S877. doi:10.1016/j.jval.2019.09.2514

48. Crimi C, Ferri S, Crimi N. Bronchiectasis and asthma: a dangerous liaison? Curr Opin Allergy Clin Immunol. 2019;19(1):46-52. doi:10.1097/ACI.0000000000000492

49. Kavanagh J, Roxas C, Thomson L, et al. P89 real-world 1 year effectiveness of benralizumab in severe eosinophilic asthma. Thorax. 2019;74(Supp12):A138-A138. doi:10.1136/thorax-2019BTSabstracts2019.232

\section{Publish your work in this journal}

The Journal of Asthma and Allergy is an international, peer-reviewed open-access journal publishing original research, reports, editorials and commentaries on the following topics: Asthma; Pulmonary physiology; Asthma related clinical health; Clinical immunology and the immunological basis of disease; Pharmacological interventions and

Submit your manuscript here: https://www.dovepress.com/journal-of-asthma-and-allergy-journal new therapies. The manuscript management system is completely online and includes a very quick and fair peer-review system, which is all easy to use. Visit http://www.dovepress.com/testimonials.php to read real quotes from published authors. 